\title{
Los determinantes de la confianza del comprador online. Comparación con el caso de subasta ${ }^{1}$
}

\section{Determinants of online buyer trust. A comparison with the auction system}

\author{
Sonia San Martín Gutiérrez \\ Carmen CAMARero IzQuierdo ${ }^{3}$
}

Recibido el 23 de noviembre de 2009, aceptado el 5 de julio de 2010

$\mathrm{N}^{\mathrm{o}}$ de clasificación JEL: M31

DOI: $10.5295 / \mathrm{cdg} .100187 \mathrm{ss}$

Reseña bibliográfica: SAN MARTÍN, S. y CAMARERO, C. (2010): "Los determinantes de la confianza del comprador online. Comparación con el caso subasta", Cuadernos de Gestión, Vol 10, n especial, pp.43-61, DOI: $10.5295 / \mathrm{cdg} .100187 \mathrm{ss}$

\section{Resumen:}

Los trabajos de investigación sobre comercio electrónico en Internet ocupan gran parte de la investigación de marketing, pero son pocos aún los que se han ocupado de estudiar el sistema de subasta. Ante las peculiares características de una compra online, transmitir confianza al consumidor en este contexto es uno de los retos más importantes y difíciles en comparación con la compra offline. No obstante, es de esperar que las señales generadoras de confianza sean diferentes en la compra online por subasta que en la compra online en otros sitios web. En este trabajo se presenta un modelo que trata de reflejar el efecto que tienen las señales del sitio web sobre la confianza del consumidor y se compara para un contexto de venta por subasta y el resto de contextos de compra online. Con la información recogida sobre una muestra de compradores online se presentan los principales resultados e implicaciones.

Palabras clave:

Comercio electrónico; subasta; confianza; calidad de servicio; garantía; políticas de privacidad y seguridad; diseño del sitio web.

\begin{abstract}
:
Studies on electronic Internet commerce are an important part of the research in marketing, bur there are still few works that have dealt with auction systems. Because of the especial characteristics of online buying, one of the most important and the most difficult challenges in the context of an online purchase as opposed to offline
\end{abstract}

\footnotetext{
${ }^{1}$ Nos gustaría poner de manifiesto nuestro agradecimiento al Ministerio de Educación y Ciencia por la financiación recibida para la realización del proyecto sobre comercio electrónico en el que se enmarca este trabajo (referencia SEJ 2007-63378).

${ }^{2}$ Profesora Titular de Universidad. Departamento de Economía y Administración de Empresas. Facultad de Ciencias Económicas y Empresariales. Universidad de Burgos. C/ Parralillos, s/n, 09001. Universidad de Burgos. E-mail: sanmargu@ubu.es
}

${ }^{3}$ Universidad de Valladolid. E-mail: $\underline{\text { camarero@ubu.es }}$ 
purchases is how to generate consumer trust. Nevertheless, it is likely that the signals that generate trust will differ in online auctions from online purchasing through other websites. This work presents a model that attempts to capture the effects of web-site signals on consumer trust and draws comparisons between auction and the rest of online shopping contexts. The main results and implications are presented using information collected from a sample of online buyers.

\section{Key Words:}

Electronic commerce; auction; trust; service quality; privacy and security policies; web site design. 


\section{INTRODUCCIÓN}

Internet ha supuesto un crecimiento sin precedentes de nuevas formas de intercambio entre consumidores, fuera de los canales de compraventa tradicionales. Entre las diferentes formas de compra a través de Internet, se encuentra la compra a una empresa en su sitio web B2C (business-to-consumer) y la compraventa entre particulares C2C (consumer-toconsumer), siendo la más conocida la modalidad de subasta tipo eBay, que en los últimos años ha sufrido un gran desarrollo (Liberos, 2010).

Tras el incremento de la investigación en el ámbito online en los últimos años, está adquiriendo interés el estudio del sistema de venta $\mathrm{C} 2 \mathrm{C}$, aunque la mayoría de trabajos se ocupan de analizar los tipos y mecanismos del sistema de subasta (Kwon et al., 2002; Park y Bradlow, 2005), de fijación de precios (Kung et al., 2002; Hou, 2007; Rice, 2007; Tu, 2008, Kumar y Maher, 2008), los comportamientos de compra mediante subasta (Weinberg y Davis, 2005; Peters y Bodkin, 2007) o los factores influyentes en la compra por subasta (Black, 2005; Finch, 2007; Shehryar, 2008). Como señalan varios trabajos recientes, es necesario estudiar más este tipo de intercambios entre particulares (Black, 2005; Jones y Leonard, 2007; Plouffe, 2008).

Precisamente el objetivo de este estudio es avanzar en el estudio comparado del comercio $\mathrm{C} 2 \mathrm{C}$ frente al comercio B2C en lo que respecta a la generación de confianza en el consumidor. Generar confianza en el consumidor es fundamental para conseguir relaciones de compraventa satisfactorias en un contexto online, sea a una empresa o a otro particular (Constantinides, 2004; Jones y Leonard, 2007; Chen et al., 2008). La necesidad de mitigar la escasez de confianza del consumidor en la comercialización por Internet ha sido considerada esencial desde que se comenzó a investigar la compraventa online (Hoffman et al., 1999; Yoon, 2002; Ha, 2004), también en un contexto de comercio electrónico P2P (Wang et al., 2008). Salo y Karjaluoto (2007) realizan una revisión del papel que desempeña la confianza en el entorno online. $\mathrm{Y}$ es que un objetivo fundamental es reducir la incertidumbre del consumidor sobre la honradez de la empresa que está detrás del sitio web y demostrar la capacidad de ésta para proveer con calidad y eficacia los productos comprados por este medio. La confianza es mencionada en investigaciones previas como una variable clave para el impulso de la comercialización de productos a través de Internet (Belanger et al., 2002, Vrechopoulos et al., 2004; Bart et al., 2005; Lee et al., 2005), capaz de generar en el consumidor un conjunto de creencias positivas sobre el futuro comportamiento de la empresa (Ganesan, 1994) que incide en la intención de compra, la satisfacción y la lealtad del consumidor (Gefen, 2000; Yoon, 2002). Sin embargo, la generación de confianza del comprador online puede depender del sistema de compraventa online y éste es precisamente el tema que analiza el presente trabajo.

Ante esta situación, nuestro trabajo pretende realizar una contribución al estudio del comercio $\mathrm{C} 2 \mathrm{C}$ analizando las diferencias que se producen en la generación de confianza a partir de las señales que envía la empresa en su sitio web en un contexto de compraventa por subasta, tipo eBay (compra online $\mathrm{C} 2 \mathrm{C}$ ), frente a la compraventa en el resto de sitios web (compra online B2C). De forma más concreta, se sugiere que la confianza se puede crear desde el ofrecimiento de una garantía, las políticas de privacidad y seguridad, la calidad de servicio y el diseño del sitio web. No nos constan estudios comparativos del desarrollo de confianza en estos dos contextos de compraventa electrónica y, tras la revisión 
realizada, sólo hemos encontrado el trabajo de Jones y Leonard (2007) que compara sus resultados para el ámbito $\mathrm{C} 2 \mathrm{C}$ y los compara con otros trabajos obtenidos para el campo B2C, pero no contemplan ni señales ni confianza.

Para acometer nuestro objetivo, en el segundo apartado se ofrece un marco teórico con las variables del modelo. Aquí justificaremos la importancia de las características del sitio web a la hora de generar confianza en el consumidor cuando compra on-line. Asimismo, el segundo apartado se ocupa de justificar las hipótesis que marcan diferencias en la generación de confianza en ambos contextos de compra online. Con las hipótesis propuestas y el planteamiento del modelo global, llegamos al tercer apartado, que se centra en los detalles del estudio empírico realizado. Finalmente, el cuarto apartado recoge las principales conclusiones, implicaciones profesionales, limitaciones y líneas futuras de continuación y mejora de este trabajo.

\section{EL COMERCIO B2C Y EL COMERCIO C2C}

Como ya se ha señalado anteriormente, el comercio B2C es el que se realiza entre empresas en el papel de vendedores y consumidores en el papel de compradores. Las páginas web de las empresas se convierten en el canal de comunicación y de distribución de bienes y servicios dirigidos al consumidor final. Por su parte, el comercio $\mathrm{C} 2 \mathrm{C}$ representa una situación de compra-venta a través de Internet y que normalmente se produce entre particulares. Otras formas de comercio electrónico C2C se producen a través de los foros y chats (Jones y Leonard, 2007). También existen plataformas online en las que se producen intercambios de ficheros entre iguales, lo que se denomina sistemas P2P (peer-to-peer). De hecho, los sistemas $\mathrm{P} 2 \mathrm{P}$ han sido una gran ayuda para el desarrollo de los intercambios de productos entre particulares (Plouffe, 2008). Sin duda alguna, la venta a través de subasta online es la forma más conocida y más exitosa de comercio C2C. No obstante, pese a que el origen del comercio $\mathrm{C} 2 \mathrm{C}$ se encuentra en las plataformas de subasta online tipo eBay, actualmente dichas plataformas también se utilizan como forma de comercio B2C (es lo que Liberos (2010) denomina modalidad de clasificados como traslación a Internet del negocio tradicional). En este caso particular, que no va a ser objeto de estudio en este trabajo, las empresas ponen a la venta sus productos con un precio fijo o mediante la admisión de pujas.

Existen similitudes del sistema de subasta con la compraventa por el resto de sitios web como son el beneficio que supone la comodidad para el comprador, los bajos costes, los inconvenientes de ser la venta virtual y anónima o la dificultad de valorar la calidad del producto antes de la compra por no poder ver, tocar o probar el producto ex-ante. Asimismo, existen algunas diferencias entre la compraventa online $\mathrm{B} 2 \mathrm{C}$ y $\mathrm{C} 2 \mathrm{C}$ como es el grado de 'conexión' que pueden sentir los consumidores al relacionarse con otros consumidores en un lugar de venta que en realidad es una 'comunidad' (Pouffe, 2008), el hecho de existir gran número de vendedores -consumidores que actúan como microempresas y que difieren mucho en la calidad ofrecida- y compradores, que intercambian de una manera más impersonal que en otros sitios web (Kim, 2005; Yen y Lu, 2008a). No obstante lo anterior, el sistema de venta entre particulares $\mathrm{C} 2 \mathrm{C}$ presenta algunos problemas mejor solventados en la compraventa particular-empresa B2C como la subida oportunista de precios, el retraso en la entrega del pedido, los problemas en el servicio al cliente post-venta o el tratamiento 
de la información privada del consumidor, que es un input esencial para el cierre exitoso de la transacción (Chen et al., 2008).

Estas diferentes características o la existencia de problemas mejor o peor solventados nos permiten intuir que las señales de confianza que envían al consumidor las plataformas de venta $\mathrm{B} 2 \mathrm{C}$ y $\mathrm{C} 2 \mathrm{C}$ no tienen el mismo efecto o relevancia para el consumidor. El conocimiento del efecto de cada tipo de señal en estos dos contextos será la base para definir qué señales han de enviar los sitios web de uno y otro perfil al consumidor para incrementar su confianza y finalmente su intención de compra.

\section{DETERMINANTES DE LA CONFIANZA HACIA EL SITIO WEB}

En situaciones de información asimétrica, la teoría de señales estudia los mecanismos que puede utilizar la empresa para indicar al mercado que sus productos son de calidad y que su comportamiento es correcto, mecanismos que sirven a su vez para que el consumidor pueda inferir la calidad de los productos y conocer a la empresa (Singh y Sirdeshmukh, 2000; Wang et al., 2008). La aplicación de esta teoría al campo electrónico es aún más importante dadas las dificultades que tienen los compradores online para conocer la calidad de productos que no pueden ver, tocar o probar antes de la compra, lo que implica gran incertidumbre ante la compra. Existen algunos estudios que han analizado estas señales que envían las empresas a través de sus sitios web (Burke, 2002; Fiore, 2002), como la publicidad, la variedad de productos (Vijayasarathy Y Jones, 2001; Dawson et al., 2003); la garantía, la seguridad y la privacidad de un sitio web, el servicio de entrega, la devolución de productos defectuosos (Burke, 2002); la usabilidad del sitio web (Ranaweera et al., 2005); la interactividad (Ranaweera et al., 2005; Yadav y Varadarajan, 2005); la estética y el diseño atractivo (Szymanski y Hise, 2000; Ranaweera et al., 2005; Yip y Dempster, 2005); o la atmósfera (Burke, 2002; Vrechopoulos et al., 2004).

Basándonos en estos estudios, describimos a continuación las señales que resultan más relevantes en el comercio electrónico (Constantinides, 2004) y en qué medida difiere su influencia sobre la confianza en contextos de compra por subasta frente al resto.

Calidad de servicio. En un contexto online, la calidad del servicio se refleja en el esfuerzo de la empresa por ofrecer el mejor servicio a través de un amplio surtido de productos, la buena relación calidad-precio, la entrega correcta y una amplia, detallada información sobre el producto o servicio o una adaptación al cliente (Trocchia y Janda, 2003). La literatura ha considerado la calidad de servicio un factor decisivo para aumentar la confianza del cliente (Singh, 2002) y ha sido contrastada esta relación calidad de servicio-confianza en un contexto online por autores como Sultan y Mooraj (2001); Chen et al. (2002); Harris y Goode (2004), Gummerus et al. (2004) y Ribbink et al. (2004). De igual forma, también se destaca la importancia de la calidad de servicio en el contexto de venta por subasta para generar credibilidad en el sitio web (Kwon et al., 2002; Jones y Leonard, 2007; Yen y Lu, 2008 a y b).

Siguiendo la teoría motivacional de Herzberg (1959) y haciendo una analogía entre satisfacción y confianza, cuando los consumidores tienen más experiencia de compra -como en la compra B2C-y la calidad de servicio ha mejorado en los sitios web, esta señal puede ser considerada un factor de higiene y no sería tan relevante para formar la confianza del 
comprador porque todos los sitios web ofrecen niveles aceptables de calidad. Sin embargo, en el caso de la compra online por subasta, la calidad de servicio puede ser un factor motivador dado que aún no se han conseguido niveles de calidad de servicio homogéneos por parte de todos los vendedores por subasta. En definitiva, cuando el consumidor realiza su compra en una empresa online (B2C) espera de ésta una elevada calidad de servicio dada la elevada difusión y las considerables mejoras que ha sufrido la venta online. Sin embargo, el nivel de calidad esperado en la compra a través de subastas C2C probablemente no sea tan alto, puesto que detrás de la venta no se encuentra una sola empresa, sino un sistema basado en la adaptación de la oferta y la demanda. Esto supone que ante un incremento similar en la calidad de servicio se genere más confianza en el comprador $\mathrm{C} 2 \mathrm{C}$ que en el comprador B2C.

Jones y Leonard (2007) comparan los resultados que obtienen en su trabajo sobre compra $\mathrm{C} 2 \mathrm{C}$ con los que han obtenido otros autores para la compra $\mathrm{B} 2 \mathrm{C}$ y encuentran que en el caso $\mathrm{C} 2 \mathrm{C}$ hay más elementos que forman la calidad de servicio e influyen en la satisfacción. Así, creemos que para la confianza también es más importante la calidad de servicio en $\mathrm{C} 2 \mathrm{C}$.

H1. El efecto positivo de la calidad del servicio sobre la confianza es mayor en la compra online por subasta que en el resto de compras online.

Garantía. La garantía indica el compromiso de la empresa, no sólo con respecto a la calidad de servicio, sino también en relación al cumplimiento de las expectativas del consumidor. En realidad, la garantía es un contrato explícito que refleja la solución a posibles contingencias futuras, como los fallos en el producto (Emons, 1988). La garantía reduce los riesgos inherentes a la compra a distancia, como en los contextos de venta directa y venta online (Tan, 1999; Wood, 2000; Wang et al., 2004). En la compra C2C por subasta, el ofrecimiento de una garantía depende del vendedor particular (por ejemplo, si ofrece una política de devolución o no) (www.eBay.es).

Es de esperar que la compra online B2C sea más objetiva y el consumidor exija más en general que la compra online C2C. Como señalan Wang et al. (2008), la compra C2C se fundamenta en recomendaciones de otros usuarios que son subjetivas en cierto grado. En el caso $\mathrm{C} 2 \mathrm{C}$, el sitio de compra incluye multitud de vendedores y compradores y se asemeja a una comunidad virtual con mayor identificación y "conexión" del comprador -un consumidor- y el vendedor -otro consumidor- (Plouffe, 2008). De acuerdo con la teoría de la identidad social, la identificación de los consumidores con una comunidad supone una implicación afectiva con el grupo. Cuando el consumidor percibe similitud con otros miembros, tratará a los otros con familiaridad y voluntariamente mostrará un compromiso con el grupo (Ellemers et al., 1999; Shen y Chiou, 2009). Esta identificación actuaría como un nexo emotivo o subjetivo que lleva a un mayor entendimiento entre consumidores que entre el consumidor y la empresa. Dado que los intercambios con las empresas son más impersonales, el consumidor buscará elementos como la garantía y las políticas de seguridad y privacidad que sustituyan el contacto con una persona y generarán más confianza en la compra online B2C que en la compra online C2C. Jones y Leonard (2007) también sugieren que se busca más la garantía en las transacciones realizadas en un contexto B2C que en un contexto $\mathrm{C} 2 \mathrm{C}$, aunque no lo contrastan empíricamente. Estos autores sostienen 
que los consumidores esperan que las empresas respondan a sus necesidades, mientras que no tienen esa expectativa cuando se relacionan con los consumidores individuales.

H2. El efecto positivo de la garantía sobre la confianza es mayor en las compras online que no siguen el sistema de subasta que en la compra online por subasta.

Políticas de privacidad y seguridad. Estas políticas constituyen probablemente el atributo que más influye en las decisiones del comprador online y es que los consumidores tienen una preocupación seria y legítima sobre la privacidad de la información que proporcionan a un vendedor. Así, los compradores que más creen en la seguridad y privacidad de datos cuando compran en un determinado sitio web tenderán a confiar en el sitio web, a proporcionar más información y a comprar online, por lo que las empresas de venta online deben comunicar la información sobre privacidad y seguridad de forma clara y accesible a los consumidores (Wang et al., 2004; Yousafzai et al., 2005). También se ha resaltado la importancia de estas políticas en el contexto P2P (peer-to-peer) (Rice, 2007) y la relación de la probabilidad de pago online con la confianza online en el sistema de subasta (Black, 2005) y de la seguridad percibida en la confianza en las transacciones online (Chellapa y Pavlou, 2002). Hay estudios que señalan que eBay se preocupa en gran medida por la privacidad y seguridad realizando grandes inversiones en hardware y software siempre que un nuevo elemento de seguridad está disponible (Light, 2003; Black, 2005).

En la compra online $\mathrm{C} 2 \mathrm{C}$ por subasta, normalmente existen más opciones de pago que en la compra online B2C, en la que es frecuente que la única forma de pago sea mediante tarjeta de crédito y online, e incluso se puede completar la transacción de la manera tradicional (Black, 2005). Este hecho puede hacer que las políticas de privacidad y seguridad sean más importantes para generar confianza en la compra online B2C.

Por otro lado, y al igual que apuntábamos para el caso de la garantía, el sentimiento de comprar dentro de una comunidad virtual permite crear vínculos de identificación y empatía entre comprador-vendedor de modo que el comprador online de más importancia a los elementos más objetivos cuando compra a una empresa que cuando compra a otro particular con quien se siente más identificado.

H3. El efecto positivo de las políticas de privacidad y seguridad sobre la confianza es mayor en las compras online que no son por subasta que en la compra online por subasta.

Diseño del sitio web. Las características de diseño del sitio web (la estructura de navegación, el contenido informativo y el estilo gráfico) también influyen en las evaluaciones de calidad del canal electrónico y en la confianza del comprador on-line (Feinberg y Kadam, 2002; Montoya-Weiss et al., 2003). De hecho, el sitio web es la única forma de contacto que tiene el establecimiento virtual con sus clientes, por lo que cuidar su apariencia y estructura es fundamental (Bramall et al., 2004; Gummerus et al., 2004). Además, la estructura de la tienda virtual y la facilidad de navegación reducen los costes de búsqueda y procesamiento de la información y minimizan el tiempo dedicado a comprar (Hoque y Lose, 1999).

Aunque es de esperar un efecto positivo del diseño del sitio web sobre la confianza del comprador online en general, también en este caso es verosímil pensar en la existencia de efectos diferentes según que el contexto de compraventa sea por subasta entre par- 
ticulares o a una empresa. Apoyándonos de nuevo en la teoría motivacional de Herzberg (1959), consideramos que el desarrollo más reciente del comercio C2C presupone una menor necesidad por parte del comprador de encontrarse con un sitio web de excelente diseño, frente al B2C en el que un buen diseño que combine navegabilidad y estilo es un requisito esperado. Como en el caso de la calidad del servicio, se puede constatar que el diseño de la mayoría de páginas B2C ya es más homogéneo y es un factor de higiene, no motivador. Por ello, el consumidor que compra a empresas se fijará más en aspectos objetivos y será más exigente en cuanto a lo que puede ofrecer una tienda electrónica en la compra B2C, mientras que el comprador C2C por subasta, se dejara influir más por aspectos emocionales y subjetivos, luego es de esperar que el diseño del sitio web incida más en su confianza.

H4. El efecto positivo del diseño del sitio web sobre la confianza es mayor en la compra online por subasta que en el resto de compras online.

Figura 1

\section{Modelo propuesto}

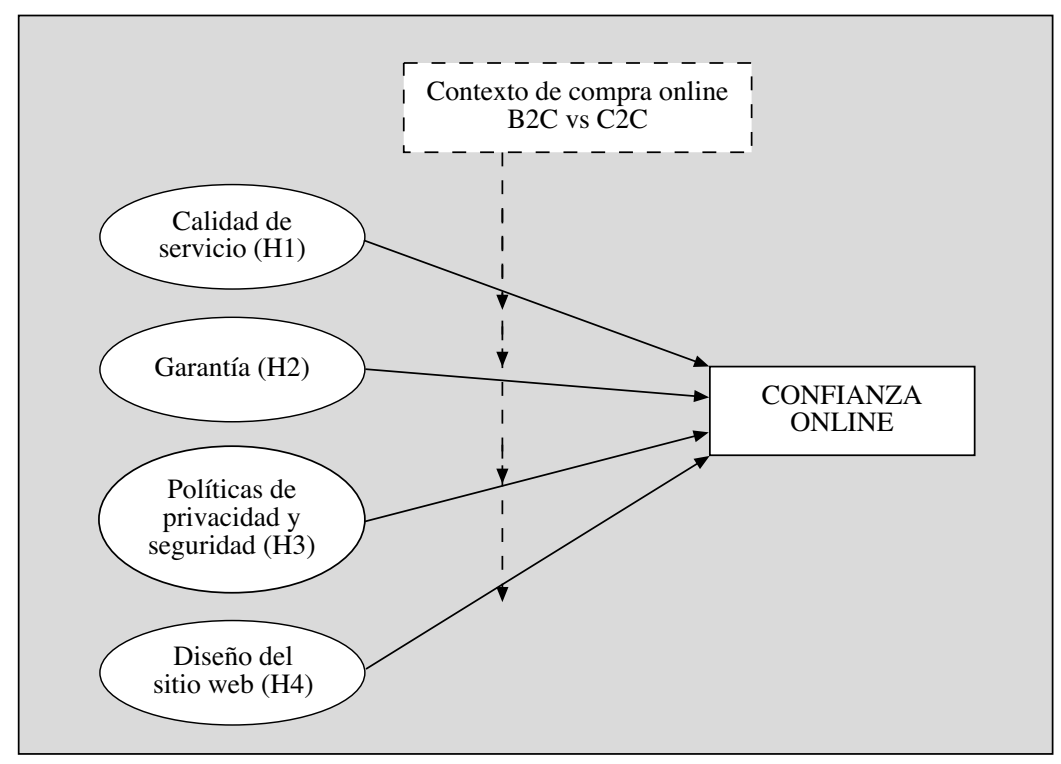

\section{ESTUDIO EMPÍRICO}

A fin de contrastar empíricamente las hipótesis planteadas se ha llevado a cabo un estudio empírico de cuyos resultados damos cuenta en el presente apartado. 


\section{1. Ámbito de estudio y muestra}

Para llevar a cabo el estudio empírico se utilizó la información recogida a través de un cuestionario realizado a compradores en Internet. Para acceder a un conjunto de población que reuniera estas características nos pusimos en contacto con los supervisores de la mayoría de los cibercentros y telecentros públicos con acceso a Internet de la comunidad de Castilla y León y con algún cibercentro de carácter privado. Los supervisores que se mostraron dispuestos a colaborar y se encargaron de distribuir el cuestionario a aquellos usuarios del cibercentro que previamente indicaran que en alguna ocasión habían comprado productos a través de Internet. Los usuarios debían indicar el nombre del sitio web en el que habían comprado en más ocasiones (comercio B2C o sitio web de subastas $\mathrm{C} 2 \mathrm{C}$ ) y valorar las características y la confianza hacia dicho sitio web y sus características. Se consideró sólo la compra de productos que pudieran adquirirse indistintamente en comercios B2C o a través de subastas $\mathrm{C} 2 \mathrm{C}$, excluyendo los casos en los que se adquirían servicios (servicios financieros y viajes fundamentalmente). Respecto a las subastas $\mathrm{C} 2 \mathrm{C}$ también quedó descartada la compra directa a empresas que venden sus productos en plataformas de subasta online (como ocurre en el caso de eBay). La recogida de información tuvo lugar en los meses de Mayo, Junio y Julio de 2008. La muestra final está formada por 104 compradores en subastas $\mathrm{C} 2 \mathrm{C}$ (en todos estos casos se señaló la dirección www.ebay.com) y 184 compradores en otras tiendas online (compra B2C). En la Tabla 1 se muestran los descriptivos de las dos muestras por sexo, edad y nivel de estudios.

Tabla 1

Características de la muestra

\begin{tabular}{lcclrllll}
\hline & Sexo & \multicolumn{3}{c}{ Edad } & \multicolumn{3}{c}{ Nivel de estudios } \\
\hline & C2C & B2C & & C2C & B2C & & C2C & B2C \\
\hline Hombre & $66 \%$ & $59.2 \%$ & Menos de 18 & $6.7 \%$ & $8.2 \%$ & Sin estudios & $1.0 \%$ & $0.6 \%$ \\
Mujer & $34 \%$ & $40.8 \%$ & $18-24$ & $56.7 \%$ & $41.8 \%$ & Educación primaria & $1.9 \%$ & $7.8 \%$ \\
& & & $25-34$ & $27.9 \%$ & $28.8 \%$ & Educación secundaria & $19.4 \%$ & $21.1 \%$ \\
& & & $45-44$ & $4.8 \%$ & $13.0 \%$ & Formación profesional & $18.4 \%$ & $17.8 \%$ \\
& & & $1.9 \%$ & $5.4 \%$ & Estudios universitarios & $59.2 \%$ & $52.8 \%$ \\
& & & $1.0 \%$ & $2.7 \%$ & & & \\
& & & & & & & & \\
\hline
\end{tabular}

\subsection{Medida de variables}

Para la medición de las variables se tomaron escalas Likert de cinco posiciones, adaptándonos en la medida de lo posible a escalas previamente validadas por otros autores para cumplir con la validez de contenido. Además realizamos 5 entrevistas en profundidad con empresas y expertos en comercio electrónico para revisar las escalas y para elaborar 
las más novedosas, como las referidas a algunas señales, en cuyo caso la mayor parte de los estudios son teóricos. Las señales o características del sitio web propuestas en este modelo son calidad del servicio, seguridad y políticas de privacidad, garantía y diseño del sitio web. La medida para la calidad de servicio fue elaborada a partir de las propuestas de Burke (2002), Montoya-Weiss et al. (2003) y Harris y Goode (2004) y para la garantía se elaboró una escala nueva. En el caso de la seguridad y privacidad se tomaron como referencia los trabajos de Harris y Goode (2004) y Montoya-Weiss et al. (2003) y finalmente para la redacción de indicadores del diseño del sitio web se siguieron los trabajos realizados por Burke (2002), Montoya-Weiss et al. (2003) y Harris y Goode (2004). Estas escalas fueron adaptadas de forma específica para este estudio con la ayuda de compañeros y las empresas entrevistadas. Dichas escalas son en su mayoría de carácter formativo, a excepción de la escala de seguridad y privacidad (véase Tabla 2). La calidad fue medida por cuatro indicadores que aluden a la información que se proporciona en la web, el cumplimiento de las condiciones, la variedad de productos y la relación calidad-precio. La seguridad y privacidad fue medida en una escala de cuatro indicadores que se refieren a la seguridad que ofrece la web en cuanto a protección de datos y seguridad en el envío de información. La garantía fue medida por un único indicador que recoge la posibilidad de devolución en caso de que el cliente no quede satisfecho. El diseño del sitio web se recoge en una escala de tres ítemes en el que se evalúa la facilidad de navegación y el diseño de la web. La confianza, por su parte, fue medida en una escala de seis indicadores basada en los ítemes propuestos por Ganesan (1994) y Doney and Cannon (1997) y adaptados al contexto online por Roy et al. (2001) y Harris y Goode (2004). Todos los ítemes aparecen en la Tabla 2.

\subsection{Validación de escalas y estimación del modelo}

Puesto que las escalas empleadas son de carácter formativo y reflectivo, optamos por la aplicación del enfoque de mínimos cuadrados parciales (PLS) para la estimación del modelo. (Chin, 1998; Chin y Newsted, 1999). Este enfoque permite trabajar con variables no normales, como es nuestro caso, y es robusto para el caso de muestras pequeñas. Para el estimación de utilizó el programa SmartPLS (Ringle et al., 2005). El nivel de significación estadística de los coeficientes se determine a través de un procedimiento de re-muestreo. El modelo se estimó por separado para cada una de las muestras. En la Tabla 2 se muestran los pesos (escalas formativas) y cargas (escalas reflectivas) factoriales y las medidas de fiabilidad. En cuanto a la validez de las escalas, para el caso de las escalas formativas, calculamos el factor de inflación de la varianza (FIV) para descartar la posible multicolinealidad de los indicadores (valores inferiores a 10). Para el caso de las escalas reflectivas (seguridad y confianza) comprobamos que todos los indicadores resultan significativos para garantizar la validez convergente, y que la correlación al cuadrado entre las variables es inferior a la varianza extraída para cada una de ellas (Tabla 3), como garantía de la validez discriminante. 
Tabla 2

Pesos y cargas factoriales y medidas de fiabilidad

\begin{tabular}{|c|c|c|c|c|}
\hline & \multirow[b]{2}{*}{ Indicadores } & \multirow[b]{2}{*}{ FIV } & \multicolumn{2}{|c|}{$\begin{array}{l}\text { Cargas/Pesos* } \\
\text { (valor de } \mathrm{t})\end{array}$} \\
\hline & & & $\begin{array}{l}\text { Compra } \\
\text { C2C }\end{array}$ & $\begin{array}{l}\text { Compra } \\
\text { B2C }\end{array}$ \\
\hline & Calidad de servicio & & & \\
\hline CAL1 & $\begin{array}{l}\text { Se proporciona información amplia y detallada sobre pro- } \\
\text { ductos y servicios que ofrece }\end{array}$ & 1.35 & $0.64(5.25)$ & $0.41(3.89)$ \\
\hline CAL2 & Ofrecen una gran variedad de productos & 1.33 & $0.10(0.63)$ & $0.02(0.12)$ \\
\hline CAL3 & $\begin{array}{l}\text { Cumplen las condiciones prometidas de calidad y plazos } \\
\text { de entrega }\end{array}$ & 1.40 & $0.30(2.23)$ & $0.40(4.34)$ \\
\hline \multirow[t]{2}{*}{ CAL4 } & $\begin{array}{l}\text { Se ofrecen productos con una buena relación calidad- } \\
\text { precio }\end{array}$ & 1.52 & $0.34(2.90)$ & $0.38(3.11)$ \\
\hline & Garantía & & & \\
\hline \multirow[t]{2}{*}{ GAR1 } & $\begin{array}{l}\text { Se ofrece una garantía que cubre posibles imprevistos o } \\
\text { fallos del producto/servicio }\end{array}$ & & $1.00(-)$ & $1.00(-)$ \\
\hline & $\begin{array}{l}\text { Seguridad y privacidad }\left(C 2 C: \alpha=0.75 ; \rho_{c}=0.83 ; \mathrm{AVE}=0.55\right) \\
\left(B 2 B: \alpha=0.83 ; \rho_{\mathrm{c}}=0.92 ; \mathrm{AVE}=0.74\right)\end{array}$ & & & \\
\hline SEG1 & $\begin{array}{l}\text { Es seguro y tiene una política de privacidad con los datos } \\
\text { de los clientes }\end{array}$ & - & $0.72(8.81)$ & $0.78(12.41)$ \\
\hline SEG2 & Se informa sobre sus políticas de seguridad y privacidad & - & $0.63(6.23)$ & $0.93(12.65)$ \\
\hline SEG3 & Me siento seguro cuando envío información personal & - & $0.83(7.34)$ & $0.98(15.89)$ \\
\hline \multirow[t]{2}{*}{ SEG4 } & Creo que se respetan mis derechos de protección de datos & - & $0.76(7.83)$ & $0.75(9.87)$ \\
\hline & Diseño del sitio web & & & \\
\hline DIS1 & Es fácil navegar & 1.23 & $0.64(4.50)$ & $0.42(2.42)$ \\
\hline DIS2 & Tiene imágenes y es entretenido comprar & 1.34 & $0.31(1.64)$ & $0.31(1.19)$ \\
\hline \multirow[t]{2}{*}{ DIS3 } & Se presenta un diseño atractivo, moderno y profesional & 1.35 & $0.43(2.25)$ & $0.69(3.99)$ \\
\hline & $\begin{array}{r}\text { Confianza }\left(C 2 C: \alpha=0.82 ; \rho_{\mathrm{c}}=0.84 ; \mathrm{AVE}=0.48\right) \\
\left(B 2 B: \alpha=0.86 ; \rho_{\mathrm{c}}=0.88 ; \mathrm{AVE}=0.56\right)\end{array}$ & & & \\
\hline CON1 & Creo que en este sitio web cumplen lo prometido & - & $0.85(8.47)$ & $0.93(13.75)$ \\
\hline $\mathrm{CON} 2$ & $\begin{array}{l}\text { Creo que la información ofrecida en este sitio web es } \\
\text { sincera y honesta }\end{array}$ & - & $0.58(7.04)$ & $0.70(11.27)$ \\
\hline CON3 & Creo que puedo fiarme de este sitio web & - & $0.68(7.94)$ & $0.79(13.31)$ \\
\hline CON4 & En este sitio web nunca se realizan falsas afirmaciones & - & $0.74(5.91)$ & $0.72(9.74)$ \\
\hline CON5 & $\begin{array}{l}\text { Este sitio web se caracteriza por su franqueza y transparen- } \\
\text { cia al ofrecer sus servicios al usuario }\end{array}$ & - & $0.62(5.92)$ & $0.67(9.75)$ \\
\hline CON6 & $\begin{array}{l}\text { Creo que este sitio web es competente para realizar su } \\
\text { trabajo }\end{array}$ & - & $0.60(6.12)$ & $0.63(8.02)$ \\
\hline
\end{tabular}

(*) Coeficientes estandarizados 
Tabla 3

Matriz de correlaciones*

\begin{tabular}{lccccc}
\hline \multicolumn{7}{c}{ Compra C2C } & & & \\
\hline & $(1)$ & $(2)$ & $(3)$ & $(4)$ & (5) \\
\hline (1) Calidad de servicio & - & & & & \\
(2) Garantía & 0,364 & - & & & \\
(3) Seguridad y privacidad & 0,478 & 0,316 & 0.741 & & \\
(4) Diseño del sitio web & 0,579 & 0,223 & 0,601 & - & \\
(5) Confianza & 0,683 & 0,474 & 0,683 & 0,554 & 0.692 \\
\hline & Compra B2C & & & \\
\hline (1) Calidad de servicio & - & & & \\
\hline (2) Garantía & 0,449 & - & & & \\
\hline (3) Seguridad y privacidad & 0,659 & 0,492 & 0.860 & & \\
\hline (4) Diseño del sitio web & 0,589 & 0,322 & 0,537 & - & \\
\hline (5) Confianza & 0,674 & 0,514 & 0,675 & 0,511 & 0.748 \\
\hline
\end{tabular}

(*) En la diagonal principal se muestra la raíz cuadrada de la varianza extraída para las escalas reflectivas.

Puesto que nuestro objetivo es comparar un mismo modelo para dos muestras diferentes es recomendable garantizar previamente la invarianza de los modelos de medida de cada grupo. Respecto a la varianza configural (ambos grupos deben compartir la misma estructura de indicadores). Tal y como se observa en la Tabla 2 todas las escalas, tanto formativas como reflectivas, mantienen una composición similar, resultando significativos los mismos indicadores. La invarianza métrica, por su parte, se refiere a la igualdad de los pesos o cargas factoriales entre grupos. En este caso nuestros datos no verifican la invarianza métrica, puesto que un test de diferencia de medias de los pesos y cargas factoriales nos arrojó diferencias significativas en un alto porcentaje de los indicadores. Pese a eso, creemos que la invarianza configural nos permite garantizar cierta estabilidad en los modelos de medida de las dos muestras y proceder a comparar los resultados de la estimación del modelo estructural. Finalmente, en la Tabla 4 se muestra la estimación de los modelos estructurales. Para comprobar la existencia de diferencias significativas entre los modelos, se aplicó un test de diferencias de medias para testar la diferencia de medias entre los parámetros.

Tabla 4

\section{Modelo structural}

\begin{tabular}{lccc}
\hline & \multicolumn{3}{c}{ Confianza } \\
\cline { 2 - 4 } & Compra C2C & Compra B2C & $\mathrm{t}$ test \\
\hline Calidad de servicio & $0.418(4.25)$ & $0.331(4.26)$ & 8.05 \\
Garantía & $0.213(3.14)$ & $0.179(3.17)$ & 4.43 \\
Seguridad y privacidad & $0.271(2.86)$ & $0.323(4.95)$ & -5.11 \\
Diseño del sitio web & $0.101(1.12)$ & $0.084(1.40)$ & 1.67 \\
\hline $\mathrm{R}^{2}$ & 0.60 & 0.58 & \\
\hline
\end{tabular}


En estos resultados observamos que la calidad de servicio es una de las variables que más repercusión tiene sobre la confianza. Además se comprueba que su influencia es mayor en el caso de que la compra se realice a través de subastas online $\mathrm{C} 2 \mathrm{C}$, por lo que no rechazamos la hipótesis H1. Respecto a H2, la garantía sí es una variable determinante de la confianza en ambos contextos, pero al contrario de lo propuesto, su efecto es superior en el contexto de compra C2C que en B2C. La seguridad y la privacidad de datos, sin embargo, sí manifiesta un efecto diferente sobre la confianza, siendo mayor dicho efecto en el caso de la compra online B2C y refutando por tanto la hipótesis H3. Por lo que se refiere al diseño del sitio web, llama la atención el hecho de que no resulte significativo en ninguno de los dos contextos de compra, lo que nos lleva a rechazar la hipótesis H4.

\section{CONCLUSIONES}

En las ventas online la creación de confianza es fundamental para conseguir un mayor desarrollo del comercio electrónico. No podemos obviar que en el contexto online es más difícil retener consumidores porque existe un gran número de alternativas, compiten además con los establecimientos físicos y la ausencia de confianza y el alto riesgo percibido desempeñan un papel de freno para la compra virtual. Por esta razón, aspectos tales como la calidad del servicio, el ofrecimiento de una garantía, de una adecuada política de seguridad y privacidad y el diseño del sitio web, pueden ser factores decisivos a la hora de ganar la confianza del consumidor en un contexto online. Dentro de este ámbito, nuestro objetivo se ha centrado precisamente en analizar si las señales que emite la empresa on-line tienen un efecto diferente sobre la confianza del cliente hacia el sitio web en función de cuál sea el medio a través del cual realiza la compra: B2C o C2C. Con ello hemos pretendido adentrarnos en un aspecto no abordado hasta el momento en la investigación en el comercio online.

Nuestra propuesta se basa en la idea de que ciertas características del sitio web como la calidad del servicio o el diseño web inciden más en la confianza en el caso del comercio $\mathrm{C} 2 \mathrm{C}$, puesto que son aspectos ya esperados por el consumidor en el caso del comercio B2C, pero pueden llegar a sorprender al cliente en un formato de comercio más reciente como es el C2C. Por otro lado, la garantía y la seguridad y privacidad de datos son más valorados en el caso del comercio B2C que en el comercio C2C, en tanto que el comprador será más exigente en seguridad cuando el vendedor es una empresa y manifestará una cierta tolerancia cuando el vendedor es otro particular.

En el estudio empírico realizado hemos comparado a los compradores online en comercios $\mathrm{B} 2 \mathrm{C}$ frente a compradores online en comercio $\mathrm{C} 2 \mathrm{C}$, en concreto en eBay, con la intención de explorar las diferencias entre ellos en la generación de confianza hacia el sitio web. Los resultados nos indican, en términos generales, que las diferencias entre estos dos grupos de compradores realmente existen, aunque no en todos los aspectos propuestos.

En primer lugar, hemos encontrado tres aspectos que influyen de forma diferente en la confianza: la calidad del servicio, la seguridad y la privacidad de datos y la garantía. El ofrecimiento de una buena calidad de servicio incrementa notablemente la confianza del comprador en los sitios de compra $\mathrm{C} 2 \mathrm{C}$, muy por encima de su influencia en otros sitios web de compra B2C. El consumidor valora especialmente que estos nuevos sistemas de compra a través de subasta garanticen un buen servicio concebido como una variedad de 
oferta, buena información, cumplimiento de plazos o una buena relación calidad-precio. Quizás el gran crecimiento que ha experimentado eBay en los últimos años guarde relación con una mejora en el servicio ofrecido y la consiguiente confianza del comprador. Algo similar ocurre con la garantía: es una señal que genera confianza, pero que resulta más relevante para los clientes $\mathrm{C} 2 \mathrm{C}$ que en clientes B2C. Aunque en ambos contextos de compra los individuos son conscientes de que la garantía en la compra es trascendental y no hay lugar a la tolerancia en este aspecto, cuando se compra en subastas la oferta de garantías es percibida como un elemento importante de diferenciación. Por último, la seguridad y la privacidad de los datos es sin duda un aspecto muy valorado en la generación de confianza. Sin embargo, los compradores en subastas online dan una importancia ligeramente inferior a esta variable, quizás debido a las múltiples opciones de pago y de entrega que permite hoy en día eBay.

En segundo lugar, hemos encontrado que el diseño del sitio web no resulta una variable determinante de la confianza en ninguno de los casos. La evidencia empírica existente sobre la influencia del diseño del sitio web en la compra online es contradictoria dado que hay autores que encuentran que los consumidores prestan más atención a aspectos superficiales del sitio, como las pistas visuales o diseño, que al contenido cuando estudiamos la confianza en el sitio web (Fogg et al., 2003), mientras que otros autores han encontrado que el diseño visual supone la menor contribución a la experiencia global con el sitio web (Sinha et al., 2001). El estudio cualitativo de Constantinides (2004) muestra que la estética del sitio web no está entre los elementos más importantes para construir una buena experiencia web, mientras que la calidad de servicio se revela como el principal factor funcional para generar interactividad con el comprador online y la seguridad como el principal factor psicológico para fomentar la confianza online (la garantía también es otro elemento psicológico para crear confianza). También en estudios previos ya se había puesto de manifiesto que el diseño del sitio web influye en la confianza indirectamente a través de la satisfacción (Autores).

En lo que se refiere a las implicaciones para la gestión, cabe resaltar que los vendedores online deben identificar los elementos clave para aumentar la confianza entre los compradores potenciales y conocer los factores capaces de reducir esa confianza (Constantinides, 2004). Y es que en el contexto de compraventa C2C, las empresas deberían ser conscientes de que tener un buen sitio web u ofrecer los productos que el comprador demanda online no es suficiente para que otro consumidor esté satisfecho con la experiencia de compra (Dawson et al, 2003; Jones y Leonard, 2007). Así, para generar confianza las empresas y servicios de venta online deben reforzar la calidad de servicio experimentada por el comprador, la garantía ofrecida y las políticas de seguridad y privacidad, en tanto que parece que los esfuerzos dedicados al diseño del sitio web no son útiles para generar confianza en el comprador online, sea cual sea el contexto de compra, por subasta o no. No obstante, existen algunas diferencias reseñables entre esos factores determinantes de la confianza en una compra $\mathrm{B} 2 \mathrm{C}$ frente una compra $\mathrm{C} 2 \mathrm{C}$, lo que hace que la creación de esta confianza dependa del tipo de compra. En los sitios de venta $\mathrm{C} 2 \mathrm{C}$ es especialmente relevante incidir en la calidad de la información facilitada al cliente, la oferta de una amplia variedad a precios competitivos y la garantía de devolución y cumplimiento de plazos. En el caso de los sitios B2C, parece existir un conjunto de factores que participan de forma equilibrada en la formación de la confianza, generando una experiencia global en el comprador. 
En cuanto a las limitaciones del trabajo, la primera sería el hecho de que la recogida de información se ha producido sólo en España, lo que impide la generalización del modelo a otros ámbitos geográficos y culturales. Tampoco se han tenido en cuenta como variables de control otras características del comprador (edad, motivo de compra, experiencia, etc.) que podrían dar lugar a diferencias en las relaciones planteadas en este estudio. Por otro lado, las características del sitio web y de la empresa han sido medidas como percepciones del consumidor, las cuales pueden no reflejar exactamente la realidad del sitio web. Además, no se han tenido en cuenta algunas particularidades de cada contexto de compra, como pueda ser la recomendación entre iguales que se da en la compra C2C o los sellos de garantía y confianza en los sitios B2C y que constituyen señales adicionales de confianza. Finalmente, se ha realizado una consideración genérica de algunos conceptos, sin matizar los diferentes componentes que engloba el diseño del sitio web o la calidad de servicio.

Estos resultados y limitaciones dan pie a plantear algunas posibles líneas futuras de investigación. Podría ser interesante estudiar la influencia de la cultura o ámbito geográfico en las percepciones, actitudes y comportamientos del comprador online (Burgman et al., 2006). También sería de interés una comparación de las señales que generan confianza y satisfacción en contextos online y offline para poder aventurar las posibles complementariedades entre ambos contextos de venta. Pese a que la lealtad offline puede ser transferida a un contexto on-line (Rafiq y Fulford, 2005), algunos autores encuentran diferencias entre ambos entornos (Shankar et al., 2003) y otros indican que es arriesgado trasladar estrategias offline a entornos virtuales (Vrechopoulos et al., 2004). Aunque existen similitudes entre ambos, también hay diferencias y particularidades en el entorno virtual, algunas de ellas relacionadas con las demandas y percepciones del consumidor. Asimismo, sería interesante plantear el estudio de la generación de confianza en el caso de otro instrumento de venta reciente, como es el teléfono móvil. Finalmente, podría ser conveniente analizar la relación entre las variables estudiadas y las características de perfil del comprador, como el sexo, la edad, la renta o la propensión a las innovaciones.

\section{REFERENCIAS BIBLIOGRÁFICAS}

BART, Y, SHANKAR, V, SULTAN, F. Y URBAN, G.L. (2005): "Are the drivers and role of on-line trust the same for all web sites and consumers? A large - Scale Exploratory empirical study", Journal of Marketing, Vol. 69, pp. 133-153.

BELANGER, F., HILLER, J.S. Y SMITH W. J. (2002): “Trustworthiness in electronic commerce: the role of privacy, security, and site attributes", The Journal of Strategic Information Systems, Vol. 11, pp. 245-270.

BLACK, G.S. (2005): "Predictors of consumer trust: likelihood to pay online", Marketing Intelligence \& Planning, Vol. 23, No. 7, pp. 648-658.

BRAMALL, C., SCHOEFER, K. Y MCKECHNIE, S. (2004): "The determinants and consequences of consumer trust in e-retailing: a conceptual framework", Irish Marketing Review, Vol. 17, pp. 13-22.

BURGMANN, I., KITCHEN, P.J. Y WILLIAMS, R. (2006): “Does culture matter on the web?", Marketing Intelligence and Planning, Vol. 24, pp. 62-76.

BURKE, R.R. (2002): "Technology and the customer interface: what consumers want in the physical and virtual store", Journal of the Academy of Marketing Science, Vol. 30, pp. 411-432. 
CHELLAPA, R.K. Y PAVLOU, P.A. (2002): "Perceived information security, financial liability and consumer trust in electronic commerce transactions", Logistics Information Management, Vol. 15 , No. $5 / 6$, pp. 358-368.

CHEN, L.-D., GILLENSON, M. L. Y SHERRELL, D. L. (2002): "Enticing online consumers: An extended technology acceptance perspective", Information and Management, Vol. 39, pp. 705719.

CHIN, W.W. (1998): "Issues and opinion on structural equation modelling”, Commentary in MIS Quarterly, Vol. 22, no 1, pp. vii-xvi.

CHIN, W.W. Y NEWSTED, P.R. (1999): "Structural Equation Modeling Analysis with small samples using Partial Least Squares". Capítulo 12, pp. 307-341. En Hoyle, R. (Ed): Statistical Strategies for small sample research, Sage Publications.

CONSTANTINIDES, E. (2004): "Influencing the online consumer's behavior: the Web experience", Internet Research, Vol. 14, No. 2, pp. 111-126.

DAWSON, L., MINOCHA, S. Y PETRE, M. (2003): "Exploring the total customer experience in e-commerce environments", Proceedings of the IADIS International Conference e-Society, pp. 945-948.

DONEY, P.M. Y CANNON, J.P. (1997): “An examination of the nature of trust in buyer-seller relationships, Journal of Marketing, Vol. 61 (Abril), pp. 35-51.

ELLEMERS, N., KORTEKAAS, P. Y OUWERKERK, J.W. (1999): "Self-categorization, commitment to the group, and group self-esteem as related but distinct aspects of social identity", European Journal of Social Psychology, Vol. 29, No. 2/3, pp. 371-89.

EMONS, W. (1988): "Warranties, moral hazard, and the lemons problem". Journal of Economic Theory, Vol. 46, pp. 16-33.

FEINBERG, R. Y KADAM, R. (2002): "E-CRM web service attributes as determinants of customer satisfaction with retail web sites", Intern(ational Journal of Service Industry Management, Vol. 13, pp. 432-451.

FINCH, B.J. (2007): "Customer expectations in online auction environments: An exploratory study of customer feedback and risk”, Journal of Operations Management, Vol. 25, pp. 985-997.

FIORE, S.G. (2002): Designing on-line experience through consideration of the salient sensory attributes of products. Tesis doctoral. UMIST. U.K.

FOGG, B.J., SOOHOO, C., DANIELSON, D.R., MARABLE, L., STANFORD, J. Y TAUBER, E.R. (2003): "How do users evaluate the credibility of Web sites?: a study with over 2,500 participants", actas de la Conference on Designing for user experiences, San Francisco (California), pp. $1-15$.

GANESAN, S. (1994): "Determinants of long-term orientation in buyer-seller relationships", Journal of Marketing, Vol. 58, pp. 1-19.

GEFEN, D. (2000): "E-commerce. the role of familiarity and trust", The International Journal of Management Science, Vol. 28, pp. 725-737.

GUMMERUS, J., LILJANDER, V., PURA, M. Y VAN RIEL, A. (2004): "Customer loyalty to content-based Web sites: the case of an online health-care service", Journal of Services Marketing, Vol. 18, pp. 175-186.

HA, H-Y. (2004): "Factors influencing consumer perceptions of brand trust online", Journal of Product and Brand Management, Vol. 13, pp. 329-342.

HARRIS, L.C. Y GOODE, M.M.H. (2004): "The four levels of loyalty and the pivotal role of trust: a study of on-line service dynamics", Journal of Retailing, Vol. 80, pp. 139-158.

HERZBERG, F., M.B. Y SNYDERMAN, B.B. (1959): The motivation to work. John Wikley. New York.

HOFFMAN, D.L., NOVAK, T.P. Y PERALTA, M. (1999): “Building consumer trust on-line”, Communications of the ACM, Vol. 42, pp. 80-86. 
HOQUE, A.Y. Y LOHSE, G.L. (1999): “An information search cost perspective for designing interfaces for electronic commerce", Journal of Marketing Research, Vol. 36, pp. 387-394.

HOU, T. (2007): "Late bidding and the auction price: evidence from eBay", Journal of Product \& Brand Management, Vol. 16, No. 6, pp. 422-428.

JONES, K. Y LEONARD, L. N. K. (2007): "Consumer-to-consumer electronic commerce: a distinct research stream", Journal of Electronic Commerce in Organizations, Vol. 5, No. 4, pp. 39-54.

KIM, Y. (2005): "The effects of buyer and product traits with seller reputation on price premiums in e-auction", Journal of Computer Information Systems, Vol. 46, No. 1, pp. 79-91.

KUMAR, S. Y MAHER, M. (2008): "Are the temptations of online reverse auctions appropriate for your Business", Supply Chain Management: an International Journal, Vol. 13, No. 4, pp. 304-316.

KUNG, M., MONROE, K. B. Y COX, J. L. (2002): "Pricing on the Internet", Journal of Product \& Brand Management, Vol. 11, No. 5, pp. 274-287.

KWON, O.B., KIM, O. Y LEE, E. J. (2002): "Impact of website information design factor son consumer ratings of web-based auction sites", Behavior and Information Technology, Vol. 21, No. 6, pp. 387-402.

LEE, B., ANG, L. Y DUBELAARC, C. (2005): "Lemons on the Web: A signalling approach to the problem of trust in Internet commerce", Journal of Economic Psychology, Vol. 26, No. 5, pp. 607-623.

LIBEROS, E. (COORD.): El libro del comercio electrónico, por r. García del poyo, j. Gil, j.A. Merino e i. Somalo. Ed. Esic. Madrid.

LIGHT, D. A. (2003): "Sure, you can trust us", Sloan Management Review, Vol. 43, p.1.

MONTOYA-WEISS, M.M., VOSS, G.B. Y GREWAL, D. (2003): "Determinants of online channel use and overall satisfaction with a relational, multichannel service provider", Journal of the Academy of Marketing Science, Vol. 31, pp. 448-458.

PARK, Y-H. Y BRADLOW, E. T. (2005): "An integrated model for bidding behavior in Internet Auctions: whether, who, when, and how much", Journal of Marketing Research, Vol. 42, No. 4 (noviembre), pp. 470-486.

PARK, J. Y STOEL, L. (2005): "Effect of brand familiarity experience and information on online apparel purchase", International Journal of Retail and Distribution Management, Vol. 33, pp. 148-160.

PETERS, C. Y BODKIN, C.D. (2007): "An exploratory investigation of problematic online auction behaviours. Experiences of eBay users", Journal of Retailing and Consumer Services, Vol. 14, pp. $1-16$

PLOUFFE, C. R. (2008): "Examining "peer-to-peer" (P2P) systems as consumer-to-consumer (C2C) exchange", European Journal of Marketing, Vol. 42, No. 11/12, pp. 1179-1202.

RAFIQ, M. Y FULFORD, H. (2005): "Loyalty transfer from offline to online stores in the UK grocery industry", International Journal of Retail \& Consumer Marketing, Vol. 33, No. 6, pp. 444-460.

RANAWEERA, C., MCDOUGALL, G. Y BANSAL, H. (2005): "A model of on-line customer behavior during the initial transaction. moderating effects of customer characteristics", Marketing Theory, Vol. 5, pp. 5-6.

RIBBINK, D., VAN RIEL, A.C.R., LILJANDER, V. Y STREUKENS, S. (2004): "Comfort your online customer: quality, trust, loyalty on the Internet", Managing Service Quality, Vol. 14, pp. 446-456.

RICE, D. O. (2007): "Protecting online information sharing in peer-to-peer (P2P) networks. A proposal for a P2P network security pricing model”, Online Information Review, Vol. 31, No. 5, pp. 682-693.

RINGLE, C. M., WENDE, S., Y WILL, A. (2005): "SmartPLS 2.0 (M3) beta", Hamburg: http:// www.smartpls.de. 
ROY, M., DEWIT, O. Y AUBERT, B. (2001): "The impact of interface usability on trust in web. Retailer",. Internet Research: Electronic Networking Applications and Policy, Vol. 11, pp. 388-398.

SALO, J. Y KARJALUOTO, H. (2007): "A conceptual model of trust in the online environment", Online Information Review, Vol. 31, No. 5, pp. 604-621.

SHANKAR, V., SMITH, A. Y RANGASWAMY, A. (2003): "Customer satisfaction and loyalty in online and off-line environments", International Journal of Research in Marketing, Vol. 20, pp. 153-175.

SHEHRYAR, O. (2008): “The effect of buyer's gender, risk-proneness, and time remaining in an Internet auction on the decision to bid or buy-it-now", Journal of Product \& Brand Management, Vol. 17, No. 5, pp. 356-365.

SHEN, C.C. Y CHIOU, J.S. (2009): "The effect of community identification on attitude and intention toward a blogging community", Internet Research, Vol. 19, No. 4, pp. 393-407.

SINGH, M. (2002): "E-services and their role in B2C e-commerce", Managing Service Quality, Vol. 12, pp. 434-446.

SINGH, J. Y SIRDESHMUKH, D. (2000): "Agency and Trust Mechanisms in Consumer Satisfaction and Loyalty Judgments", Journal of the Academy of Marketing Science, Vol. 28, No. 1, pp. 150-167.

SINHA, R., HEARST, M., IVORY, M., Y DRAISIN, M. (2001): “Content or graphics? An empirical analysis of criteria for award-winning websites", en actas del 7th Conference on Human Factors and the Web, Madison, WI, junio, extraído el 25 de septiembre de 2003 from [http://webtango. berkeley.edu/papers/hfw01/Sinha_hfw01.pdf.-

SULTAN, F. Y MOORAJ, H.A. (2001): "Designing a trust-based E-business strategy", Marketing Management, (November/December), pp. 40-45.

SZYMANSKI, D. M., Y HISE, R.T. (2000): “E-Satisfaction. An Initial Examination”, Journal of Retailing, Vol. 76, pp. 309-322.

TAN, S.J. (1999): "Strategies for reducing consumers' risk aversion in Internet shopping”, Journal of Consumer Marketing, Vol. 16, pp. 163-180.

TEO, T.S.H. Y LIU, J. (2007): "Consumer trust in e-commerce in the United States. Singapore and China", Omega, Vol. 35, No. 1, pp. 22-38.TROCCHIA, P.J. y JANDA, S. (2003): "How do consumers evaluate Internet retail service quality?", Journal of Services Marketing, Vol. 17, pp. 243-253.

TU, Y. (2008): “An application of web-based data mining: selling strategies for online auctions", Online Information Review, Vol. 32, No. 2, pp. 147-162.

VIJAYASARATHY, L.R. Y JONES, J.M. (2001): “Do Internet shopping aids make a difference? An empirical investigation”, Electronic Markets, Vol. 11, pp. 75-83.

VRECHOPOULOS, A.P., O'KEEFE, R.M., DOUKIDIS, G.I. Y SIOMKOS, G.J. (2004): "Virtual store layout. an experimental comparison in the context of grocery retail", Journal of Retailing, Vol. 80, pp. 13-22.

WANG, S., BEATTY, S.E. Y FOXX, W. (2004): "Signaling the trustworthiness of small online retailers", Journal of Interactive Marketing, Vol. 18, No. 1, pp. 53-69.

WANG, Y., WONG, D.S., LIN, K-J. Y VARADHARAJAN, V. (2008): "Evaluating transaction trust and risk levels in peer-to-peer e-commerce environments", Information Systems and E-business Management, Vol. 6, No. 1, pp. 25-48.

WEINBERG, B.D. Y DAVIS, L. (2005): "Exploring the WOW in online-auction feedbac",. Journal of Business Research, Vol. 58, pp. 1609-1621.

WOOD, S.L. (2000): "Remote purchase environments: the influence of return policy leniency on two-stage decision process", Journal of Marketing Research, Vol. 38, pp. 157-169.

YADAV, M..S. Y VARADARAJAN, R. (2005): "Understanding product migration to the electronic marketplace: a conceptual framework", Journal of Retailing, Vol. 81, pp. 125-140. 
YEN, C-H. Y LU, H-P (2008a): "Factors influencing online auction repurchase intention", Internet Research, Vol. 18, No. 1, pp. 7-25.

YEN, C-H. Y LU, H-P (2008b): "Effects of e-service quality on loyalty intention: an empirical study in online auction", Managing Service Quality, Vol. 18, No. 2, pp. 127-146.

YIP, G. Y DEMPSTER, A. (2005): "Using the Internet to enhance global strategy”, European Management Journal, Vol. 23, pp. 1-13.

YOON, S.J. (2002): "The antecedents and consequences of trust in on-line purchase decisions", Journal of Interactive Marketing, Vol. 12, pp. 47-63.

YOUSAFZAI, S.Y., PALLISTER, J.G. Y FOXALL, G.R. (2005): "Strategies for building and communicating trust in electronic banking: a field experiment", Psychology and Marketing, Vol. 22, pp. 181-201. 
